\title{
Detection of Escherichia coli O157:H7 by multiplex PCR and their characterization by plasmid profiling, antimicrobial resistance, RAPD and PFGE analyses
}

\begin{abstract}
Twenty-five and three strains of Escherichia coli O157:H7 were identified from 25 tenderloin beef and three chicken meat burger samples, respectively. The bacteria were recovered using the immunomagnetic separation procedure followed by selective plating on sorbitol MacConkey agar and were identified as E. coli serotype O157:H7 with three primer pairs that amplified fragments of the SLT-I, SLT-II and H7 genes in PCR assays. Susceptibility testing to 14 antibiotics showed that all were resistant to two or more antibiotics tested. Although all 28 strains contained plasmid, there was very little variation in the plasmid sizes observed. The most common plasmid of $60 \mathrm{MDa}$ was detected in all strains. We used DNA fingerprinting by randomly amplified polymorphic DNA (RAPD) and pulsed-field gel electrophoresis (PFGE) to compare the 28 E. coli O157:H7 strains. At a similarity level of 90\%, the results of PFGE after restriction with XbaI separated the E. coli O157:H7 strains into 28 single isolates, whereas RAPD using a single 10-mer oligonucleotides separated the E. coli O157:H7 strains into two clusters and 22 single isolates. These typing methods should aid in the epidemiological clarification of the E. coli O157:H7 in the study area.
\end{abstract}

Keyword: Antibiotic resistance; Escherichia coli O157:H7; IMS; PFGE; Plasmid; RAPDPCR 